\title{
A hybrid open-top light-sheet microscope for multi- scale imaging of cleared tissues
}

\author{
Adam Glaser ( $\nabla$ adamkglaser@gmail.com ) \\ University of Washington https://orcid.org/0000-0003-3558-8994 \\ Kevin Bishop \\ University of Washington https://orcid.org/0000-0001-8722-6464 \\ Lindsey Barner \\ University of Washington \\ Etsuo Susaki \\ Juntendo University \\ Shimpei Kubota \\ University of Tokyo \\ Gan Gao \\ University of Washington \\ Robert Serafin \\ University of Washington
}

Pooja Balaram

Allen Institute for Brain Science

Emily Turschak

Allen Institute for Brain Science

Philip Nicovich

Cajal Neuroscience

Hoyin Lai

SVision Technologies LLC

Luciano Lucas

SVision Technologies LLC

Yating Yi

Texas A\&M University

Eva Nichols

University of Washington

Hongyi Huang

University of Washington

Nicholas Reder

University of Washington

Jasmine Wilson 
University of Washington

\section{Ramya Sivakumar}

University of Washington

\section{Elya Shamskhou}

University of Washington

\section{Caleb Stoltzfus}

University of Washington https://orcid.org/0000-0002-0543-345X

\section{Xing Wei}

University of Washington

\section{Andrew Hempton}

University of Washington

\section{Marko Pende}

MDI Biological Laboratory

\section{Prayag Murawala}

MDI Biological Laboratory https://orcid.org/0000-0002-0607-1059

\section{Hans Dodt}

Vienna University of Technology

\section{Takato Imaizumi}

University of Washington

\section{Jay Shendure}

University of Washington https://orcid.org/0000-0002-1516-1865

\section{Brian Beliveau}

University of Washington https://orcid.org/0000-0003-1314-3118

\section{Michael Gerner}

University of Washington https://orcid.org/0000-0001-5406-8308

\section{Li Xin}

University of Washington

\section{Hu Zhao}

Texas A\&M University

\section{Lawrence True}

University of Washington

\section{R. Clay Reid}

Allen Institute for Brain Science https://orcid.org/0000-0002-8697-6797

\section{Jayaram Chandrashekar}

Howard Hughes Medical Institute https://orcid.org/0000-0001-6412-0114

\section{Hiroki Ueda}

The University of Tokyo

\section{Karel Svoboda}

Janelia Research Campus, Howard Hughes Medical Institute https://orcid.org/0000-0002-6670-7362 
Jonathan Liu

University of Washington https://orcid.org/0000-0001-5650-3086

\section{Article}

Keywords: multi-scale imaging, microscopy, cleared tissues

Posted Date: September 3rd, 2021

DOl: https://doi.org/10.21203/rs.3.rs-860253/v1

License: (c) (1) This work is licensed under a Creative Commons Attribution 4.0 International License. Read Full License

Version of Record: A version of this preprint was published at Nature Methods on May 11th, 2022. See the published version at https://doi.org/10.1038/s41592-022-01468-5. 


\section{Abstract}

Light-sheet microscopy has emerged as the preferred means for high-throughput volumetric imaging of cleared tissues. However, there is a need for a user-friendly system that can address imaging applications with varied requirements in terms of resolution (mesoscopic to sub-micrometer), sample geometry (size, shape, and number), and compatibility with tissue-clearing protocols and sample holders of various refractive indices. We present a 'hybrid' system that combines a novel non-orthogonal dual-objective and conventional (orthogonal) open-top light-sheet architecture for versatile multi-scale volumetric imaging.

\section{Main Text}

Recent advances in tissue-clearing protocols greatly reduce optical scattering, aberrations, and background fluorescence, enabling deep-tissue imaging with high resolution and contrast. These approaches have yielded new insights in many fields, including neuroscience, developmental biology, and anatomic pathology [1-11]. Light-sheet microscopy has emerged as a preferred means for highresolution volumetric imaging of cleared tissues due to its unrivaled speed and low photobleaching [12, 13]. Many variants of light-sheet microscopes have been developed in recent years by academic researchers and commercial entities to tackle a diverse range of imaging applications (Error! Reference source not found. and Error! Reference source not found.) [14-18]. Whereas individual light-sheet systems are well-suited for a subset of cleared-tissue applications, trade-offs are inevitable. In particular, no current light-sheet microscope can satisfy all of the following requirements: (1) user-friendly mounting of multiple specimens with standard holders, (2) compatibility with all current clearing protocols, (3) no fundamental limits on lateral specimen size, (4) a large imaging depth to accommodate intact mouse organs and thick tissue slabs, and (5) a wide range of optical resolutions for time/data-efficient imaging from sub-micrometer to mesoscopic scales (i.e., 'multi-scale' imaging). A system that meets these diverse requirements would satisfy a growing base of researchers interested in rapidly screening large intact cleared tissues followed by detailed interrogation of sub-micrometer structures within localized subregions.

The majority of cleared-tissue microscopes use an illumination objective that is oriented in the horizontal plane of the specimen, along with a collection objective that is orthogonal to the illumination objective, i.e., an orthogonal dual-objective (ODO) configuration $[16,19]$ (Fig. 1a). This imaging geometry places physical constraints on the lateral size of the specimen such that it is not possible to image large centimeter-scale tissue slabs or multiple specimens mounted in standard holders (e.g., well plates). Therefore, we and others have previously developed ODO systems with an inverted geometry, which overcomes these constraints by tilting both objectives and placing them above or below the specimen [20-27] (Fig. 1b). In particular, open-top light-sheet (OTLS) microscopy systems provide an ease-of-use similar to a flat-bed document scanner by placing all optical components below the sample holder [2227]. This enables a wide range of modular sample holders to be used (e.g. well plates), as well as potential accessory technologies such as microfluidics, electrophysiology, and microdissection/aspiration. With OTLS systems, the angled orientation of the objectives reduces the usable 
imaging depth of the systems to well below each objective's native working distance. In addition, in OTLS microscopy, angling the optical paths with respect to a horizontal sample holder can introduce significant off-axis aberrations (especially for higher-numerical-aperture beams) unless the refractive index of the specimen and the sample holder are exquisitely well-matched (Supplementary Fig. 1). This can reduce the ease of use and versatility of high-numerical-aperture (high-NA) OTLS systems.

To overcome these issues, we considered the use of a single-objective architecture that has gained popularity in recent years, in which the illumination and collection beams share a single objective [28-40] (Fig. 1c). For an open-top version of single-objective light-sheet microscopy, orienting the objective in the vertical direction with respect to a horizontal specimen holder makes use of the objective's full working distance and dramatically increases the system's tolerance to refractive-index mismatch (Supplementary Fig. 2). However, single-objective OTLS microscopy is incompatible with multi-scale imaging because, to the best of our knowledge, no currently available objective provides both sub-micrometer resolution and a large mesoscopic field of view (FOV). In addition, the use of a single objective both constrains and couples the illumination and collection beams such that there is less flexibility to tailor the axial and lateral resolutions (Supplementary Fig. 3) [41]. Recently, we have explored the concept of a nonorthogonal dual-objective (NODO) OTLS configuration that makes use of a high-NA collection objective oriented in the vertical direction (similar to single-objective OTLS microscopy) in conjunction with a separate angled objective to provide lower-NA light-sheet illumination (Fig. 1d). Our simulations indicate that a NODO configuration maintains the high index-mismatch tolerance of single-objective OTLS microscopy (Supplementary Fig. 4) and offers increased flexibility to optimize lateral and axial resolutions [41].

To address the varied requirements of cleared-tissue light-sheet microscopists, we designed a 'hybrid' OTLS microscope that is the first system to use a non-orthogonal dual-objective (NODO) configuration (for high-resolution imaging). For low-resolution imaging, a conventional ODO open-top system is integrated with the NODO system. This new hybrid system leverages the strengths and overcomes the limitations of previous systems (Supplementary Note 1), addressing the five requirements listed earlier: (1) simple open-top mounting of multiple specimens in standard holders such as well plates, (2) the ability to pair any tissue-clearing reagent with nearly any sample-holder material with negligible degradation in imaging performance (i.e., high tolerance to refractive-index mismatch, Supplementary Fig. 5), (3) no lateral constraints on the specimen size (limited only by the travel range of the microscope stage), (4) a 1-cm imaging depth for comprehensive interrogation of intact mouse organs and thick tissue slabs, and (5) multi-scale imaging over an unprecedented range roughly corresponding to what is achieved with $2 X$ to $40 X$ objectives. These unique capabilities open the door for new light-sheet microscopy applications, including efficient multi-scale imaging workflows in which one or more large specimens must be rapidly screened at low resolution to identify localized regions of interest for quantitative interrogation at the sub-micrometer scale.

The layout of our new system is shown in Fig. 1e. The system architecture features three main objectives that are selected to avoid geometric interference. All three objectives are positioned below the specimen, 
which provides an unobstructed open top that enables volumetric imaging over a large $12 \times 7.5 \times 1 \mathrm{~cm}$ $(x y z)$ imaging volume. The imaging volume is limited in $z$ by the objectives and in $x y$ by the mechanical limits of the motorized stage. All three objectives are sealed into a monolithic imaging chamber through direct immersion or the use of a solid-immersion meniscus lens (SIMlens), which provides multiimmersion capabilities spanning the refractive index range of all current clearing protocols (Supplementary Fig. 6) [26, 27].

The optical layout of the system is shown in Fig. 1f. To achieve an optimal combination of submicrometer resolution, large imaging depth, and compatibility with standard sample holders, we developed a new NODO light-sheet configuration [41]. By using the full numerical aperture (NA) of a vertically oriented objective for fluorescence collection, and a separate objective for non-orthogonal illumination, our new NODO architecture provides superior resolution to that of a single-objective lightsheet system (if based on the same primary objective) and relaxes the NA requirements for the remotefocus module that is necessary for these non-orthogonal light-sheet systems (Supplementary Fig. 4). This allows for the use of a wider range of moderate-NA primary (collection) objectives for cleared-tissue imaging, as well as simple air objectives at the remote-focus module rather than bespoke objective assemblies (Supplementary Fig. 7). We carefully selected our NODO collection objective and its optical and mechanical specifications to enable the placement of a separate low-NA collection objective oriented orthogonally to the illumination objective (Supplementary Fig. 8). This forms an additional ODO imaging path for rapid mesoscopic-resolution imaging. Similar to single-objective light-sheet systems, the NODO path uses a remote focus to re-image the non-orthogonal light sheet onto a camera, whereas the ODO collection path directly images the orthogonal light sheet onto a camera (Supplementary Figs. 9-16 and Supplementary Video 1 contain optical models, a photograph of the hybrid OTLS microscope, and additional point spread functions for both imaging paths). The same illumination path is used to generate a light sheet with a variable width for both the NODO and ODO imaging paths.

The overall magnification of each imaging path is tunable, limited at the extremes by either the NA or FOV of the collection objectives (see Supplementary Note 2 for further discussion of these two imaging modes), corresponding to a combined lateral-resolution range of $\sim 0.4-10 \mu \mathrm{m}$, and volumetric imaging speeds of $\sim 5 \mathrm{~mm}^{3}$ to $10 \mathrm{~cm}^{3}$ per hour (Fig. $1 \mathrm{~g}$ ). In the current microscope configuration, the NODO path provides an XYZ resolution of $0.45 \pm 0.07,0.46 \pm 0.05$, and $2.91 \pm 0.31 \mu \mathrm{m}(N=437$ beads), and the ODO path provides an XYZ resolution of $4.41 \pm 0.83,4.09 \pm 1.07$, and $5.48 \pm 1.08 \mu \mathrm{m}$ ( $N=109$ beads) (mean \pm standard deviation, Fig. 1h). Representative imaging results of a CUBIC-cleared mouse brain labeled with brain-wide arterial (ASMA) and nuclear (SYTOX-G) stains are shown in Figs. 1i-k (Supplementary Video 2). The entire intact specimen is rapidly imaged with near-isotropic resolution using the mesoscopic ODO path of the hybrid system, which clearly resolves vasculature in all three dimensions. Detailed interrogation of a sub-region with the high-resolution NODO path resolves individual smooth muscle cells and sub-nuclear features, which are not resolved in ODO images of the same sub-region. We further spotlight the unique utility of our hybrid system in two example applications where multi-scale imaging enables time- and data-efficient experimental workflows. 
First, we imaged axons in an intact mouse brain cleared in ethyl cinnamate (ECi) (Supplementary Video 3). Tracking the axons of individual neurons is a challenging problem - axons can be very thin (100 nm) and span very large distances $(\mathrm{cm})$. To do this effectively, one typically relies on sparse and bright labeling of a few neurons along with high-resolution, high-contrast imaging of the entire brain $\left(\sim 0.5 \mathrm{~cm}^{3}\right)$ $[42,43]$. Sub-micrometer imaging of such large volumes generates data sets that are tens of terabytes in size, necessitating computationally intensive downstream pipelines for data handling, processing, and storage. The multi-scale imaging capability of our hybrid system greatly accelerates and simplifies this process by screening an entire brain at low resolution to identify target regions with imageable neurons, followed by high-resolution imaging of the identified regions of interest.

Using the ODO imaging path, a mouse brain was rapidly screened in $\sim 1$ hour with isotropic $\sim 2$ - $\mu \mathrm{m}$ voxels

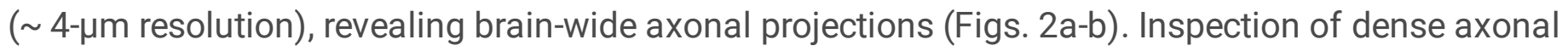
projections in $x y$ and $x z$ planes through the midbrain confirms the ability of the system to provide nearisotropic 4- to 5- $\mu \mathrm{m}$ resolution throughout the entire intact brain (Fig. 2c). A targeted region of interest around a cortical pyramidal neuron was then imaged at $<0.5$-um $x y$ resolution using the NODO imaging path (Fig. 2d-e). This imaging resolution is sufficient to discern spines and varicosities on individual dendrites and axons (Figs. 2f-h). Importantly, imaging a 1- $\mathrm{mm}^{3}$ subregion containing the targeted neuron at $\sim 0.5 \times 0.5 \times 2.7 \mu \mathrm{m}(x y z)$ resolution required only 24 minutes, generating a 180-GB data set. By comparison, imaging the entire $0.5-\mathrm{cm}^{3}$ brain volume at this resolution would require $\sim 2$ weeks of imaging and would produce 200 TB of imaging data.

In a second example of the unique capabilities of our hybrid OTLS system, an imaging experiment was performed that required broad multi-scale imaging across multiple specimens. We used our hybrid OTLS system to study metastatic colonies from two cancer cell lines (MDA-231 and OS-RC-2) throughout intact mouse brains (Supplementary Video 4). Due to the sparse and unpredictable spatial distribution of brain metastases, identifying those sites in whole brains is challenging without a rapid low-resolution screening method. Once metastatic sites are identified, high-resolution quantitative analysis of these regions is also desired. While past studies have relied on laborious and time-consuming experiments with manual transfer of individual specimens between different microscope systems [44] (for low-resolution localization followed by high-resolution analysis), we analyzed six intact mouse brains in a single imaging session without manually removing or remounting the specimens (Fig. 3a).

All mouse brains were first placed in a standard 6-well plate (Fig. 3b) and screened sequentially using fast mesoscopic ODO imaging (Fig. 3c). After imaging, 34 total regions of interest (ROI) centered on metastatic colonies were manually identified across all mouse brains and sequentially imaged without having to move the specimens or specimen holder. An example ROI from each brain is shown in Fig. 3d, with all 34 ROIs shown in Supplementary Fig. 17. Visual inspection of whole-brain images reveals that metastatic growth from MDA-231 cancer cells exhibit vessel co-option (Figs. 3e-g) whereas OS-RC-2 cancer-cell metastases do not. Morphological analysis of volumetrically segmented brain metastases, performed across the high-resolution ROIs, reveal statistically significant differences between the two metastatic cell lines. For example, the "volume per surface area" shape metric, which correlates with the 
roundness of a metastatic colony, was found to be higher for the OS-RC-2 cell line (Fig. 3i). In addition, for the MDA-231 cancer cell line, the density of cancer cells in the vessel co-option ROls was found to be lower than the adjacent metastatic colonies (Fig. 3j). Both of these findings are consistent with the previous report from Kubota et al [44]. However, while this previous study required two separate microscope systems and several weeks of tedious imaging, including manual specimen transfer and coregistration of coordinates (personal correspondence with S.I.K.), our hybrid OTLS system allowed the entire imaging experiment to be completed in $\sim 1$ day ( 2 hours of low-resolution imaging time per whole brain and $\sim 20$ minutes of high-resolution imaging time per ROI).

Compared to existing academic and commercial light-sheet microscopy systems, our hybrid OTLS system provides a unique combination of versatility and performance necessary to satisfy the diverse requirements of a growing number of cleared-tissue imaging applications. In addition to the imaging examples in Figs. 1-3, the system has potential utility for multi-scale non-destructive 3D pathology of prostate cancer with ECi clearing (Supplementary Fig. 18), whole brain imaging of endogenous fluorescent proteins with PEGASOS clearing (Supplementary Fig. 19 and Supplementary Video 5), 3D imaging of mouse embryos with SHIELD clearing (Supplementary Fig. 20), mapping of immune cell populations in 3D with Ce3D clearing (Supplementary Fig. 21 and Supplementary Video 6), assessment of 3D cell proliferation with iDISCO clearing (Supplementary Fig. 22), imaging of plants/pigmented animal models processed with ClearSee/DEEP-Clear (Supplementary Fig. 23), screening of immunofluorescent and endogenously fluorescent mouse brains with CUBIC clearing (Supplementary Fig. 24 Supplementary Videos 7-9), and large-scale imaging of amyloid plaques in thick human brain slices measuring up to $12 \times 7.5 \times 1 \mathrm{~cm}$ in size (Supplementary Fig. 25 and Supplementary Video 10).

In the future, both the axial and lateral resolution of the hybrid OTLS system can be improved. For example, the axial resolution of the system can be enhanced by incorporating a Bessel beam or by using a higher-NA Gaussian beam for light-sheet illumination $[17,18,45,46]$ (Supplementary Fig. 26). This is facilitated by the fact that unlike single-objective light-sheet systems, our new NODO architecture allows the illumination NA to be substantially increased without necessitating a concomitant decrease in the collection NA (i.e. decoupled optical paths as shown in Fig. 1d). With the 45-deg orientation of the two low-NA objectives in our system (one on either side of the NODO collection objective), it may also be possible to perform dual-sided illumination with fusion deconvolution to achieve improved image quality (Supplementary Fig. 27) [20, 37, 47]. The lateral resolution of the system can also be improved by increasing the collection NA of the multi-immersion objective. However, the optical cone angle cannot be increased beyond 40-45 deg for a hybrid NODO / ODO system, as this would prevent the ODO objectives from being positioned at $45 \mathrm{deg}$ with respect to the vertical axis.

While our current design uses three separate objectives, it is theoretically possible to achieve improved performance with an optimized NODO objective (Supplementary Fig. 28), or to achieve similar multi-scale OTLS performance using a single high-NA objective with a large FOV and working distance (see Supplementary Note 4 and Supplementary Fig. 29). For example, the pupil of a single objective could be split into three separate regions, using the edges for NODO illumination and for mesoscopic ODO 
imaging, and only the center for high-resolution NODO collection. However, this would require an objective with extraordinary specifications that could be prohibitively expensive to design and manufacture.

Therefore, in summary, our hybrid OTLS design represents a practical means, with commercially available optical components, of achieving an impressive balance of performance and versatility for a growing number of cleared-tissue imaging experiments in which rapid low-resolution screening of large volumes is desired in addition to high-resolution characterization of localized subregions.

\section{Online Methods}

\section{Hybrid open-top light-sheet microscope}

Light-sheet-based imaging is achieved using three optical arms. The first obliquely illuminates the specimen with a light sheet at 45 deg relative to the vertical axis. The second is oriented vertically, enabling high-resolution collection of the light-sheet-generated fluorescence in a NODO configuration. Finally, the third arm is oriented at 90 deg relative to the illumination light sheet, enabling high-speed mesoscopic imaging in an ODO configuration. All three objectives are positioned below the specimen and are sealed into a custom-designed monolithic imaging chamber (machined by Hilltop Technologies). In the case of each angled air objective, a custom-fabricated solid-immersion meniscus lens (SIMlens) (fabricated by BMV Optical Technologies) is sealed into the chamber (Supplementary Fig. 6) [26]. Stagescanning is achieved by using an XY stage (MS2000, Applied Scientific Instrumentation) attached to two Z-axis risers (LS50, Applied Scientific Instrumentation). 405, 488, 561, and $638 \mathrm{~nm}$ excitation laser light is provided to the illumination path by a multi-line laser package (Cobolt Skyra, HÜBNER Photonics). The NODO collection path is equipped with a motorized filter wheel (FW102C, Thorlabs) with four filters that can be used with 405-nm (FF02-447/60 - 25, Semrock), 488-nm (FF03-525/50 - 25, Semrock), 561-nm (FF01-618/50 - 25, Semrock), or 638-nm (BLP01-647R-25, Semrock) excitation. The ODO collection path is equipped with a single multi-band-pass filter (FF01-432/515/595/730 - 25, Semrock) that can be used with any of the four excitation wavelengths. The entire system is compact and fits on a portable 2' $\times 3^{\prime}$ optical cart (POC001, Thorlabs).

\section{Illumination optical path}

The illumination optical arm is shown in Supplementary Fig. 9. The illumination optics are designed to allow for the light-sheet properties (i.e., width, thickness, and depth of focus) to be adjusted. In addition, the optical path is designed to be compatible with the refractive index, $n$, of all current clearing protocols (i.e., it has multi-immersion capabilities) and to minimize chromatic aberrations and defocusing (i.e., variations in the illumination focal length as a function of wavelength).

Illumination light is fiber coupled into the system with a Gaussian numerical aperture (NA) of $\sim 0.12$ and collimated using an objective (RMS20X, Olympus). The beam diameter is then adjusted using a $4 \mathrm{X}$ variable beam expander (BE052-A, Thorlabs). This serves to adjust the overall NA of the light sheet. The variably expanded beam is then passed through an electronically tunable lens (ETL) that enables axial adjustment/alignment of the light sheet (EL-16-40-TC-VIS-5D-C, Optotune). The axially adjusted beam is 
relayed 1:1 using a pair of lenses (AC254-75A, Thorlabs) so that it can be scanned using a pair of largebeam-diameter galvanometric scanning mirrors (GVS012, Thorlabs). One mirror is scanned to create a digitally scanned light sheet [48]. The other scanning mirror is used to align the light sheet with the focal plane of the NODO and ODO imaging paths. A pair of achromatic doublet lenses (AC508-75A and AC508$200 \mathrm{~A}$, Thorlabs) are then used to relay the scanned beam to the back focal plane of a $2 \mathrm{X}$ illumination objective with NA $=0.10$ (TL2X-SAP, Thorlabs). Finally, the illumination light travels through the SIMlens (Supplementary Fig. 6) [26]. The SIMlens provides multi-immersion performance and prevents aberrations (spherical, off-axis, and chromatic) of the light sheet by minimizing refraction of the illumination rays as they transition from air into the immersion medium. In addition, since ray angles are preserved as they transition between air and the immersion medium, the SIMlens increases the NA of the illumination light sheet by a factor of $n$. When combined, this optical design yields a light sheet with tunable NA $(0.025-0.10 \times n)$ and tunable width $(0-11 \mathrm{~mm} / n)$ limited by the field of view (FOV) of the illumination objective.

A digitally scanned light sheet was chosen over a cylindrical-lens approach (static light sheet), as this facilitates achieving a high level of tunability for multi-scale imaging. Moreover, large scanning mirrors were selected to fill the back focal plane of the final illumination objective and to avoid having to significantly magnify the beam after the scanning mirrors, which would reduce the lateral scanning range of the mirrors (i.e., constrain the maximum light-sheet width). In the current design, rotating the scanning mirror results in lateral scanning at a ratio of $\sim 0.60 \mathrm{~mm}$ per deg / $n$. The maximum desired light sheet width (i.e., lateral scanning range) is $11 \mathrm{~mm}$, corresponding to a scanning angle of $\sim 18 \mathrm{deg}$, which is within the maximum scan range of the galvo scanner (20 deg).

\section{NODO optical path}

The physical layout, ZEMAX model, and objective options for this NA-maximized NODO imaging configuration are shown in Supplementary Fig. 10. The NODO optical path of our system uses a multiimmersion objective (\#54-12-8, Special Optics) with a long 1-cm working distance. This objective is compatible with all clearing protocols $(n=1.33-1.56)$ and provides a NA of 0.483 (in air) that scales with the index of the immersion medium (e.g., NA 0.75 at $n=1.56$ ). The lens is oriented in the normal (vertical) direction with respect to the specimen holder/interface and is therefore non-orthogonal to the light sheet. To image this non-orthogonal light sheet, we use a remote-focus imaging strategy analogous to what is used for single-objective light-sheet systems, with the multi-immersion objective serving as the primary objective $(01)[28,29]$.

To minimize aberrations in the remote focus relay, the overall magnification from the specimen to the remote focus (air) should be equal to the refractive index of the specimen, $n$. Given this requirement, the relay lenses and first remote objective (02) must be carefully selected. For 02 , a $20 \mathrm{X}$ objective is optimal, as a 10X or 40X objective would clip either the NA or FOV of our 01. Of the several companies that produce microscope objectives, Zeiss and Leica were avoided because chromatic aberrations are partially corrected in the tube lenses produced by these companies, which would complicate selection of the two relay tube lenses. Therefore, only objectives from Olympus and Nikon were considered, where 20X 
objectives from Olympus have a focal length of $9 \mathrm{~mm}$, and 20X objectives from Nikon have a focal length of $10 \mathrm{~mm}$. Factoring in the effective focal length of $01(12.19 \mathrm{~mm} / \mathrm{n})$, the required relay lens magnification is $\sim 1.219 \mathrm{X}$ for Nikon and $\sim 1.354 \mathrm{X}$ for Olympus. Note that the magnification of the multiimmersion objective in our system scales inversely as a function of $n$. This allows us to use a fixed set of relay lenses and always satisfy the remote focusing magnification requirement. However, this would be problematic with alternative multi-immersion objectives, where the effective focal length does not vary with $n$, and therefore the magnification of the relay lenses would need to be adapted for each immersion medium.

Well-corrected tube lenses are available with a limited selection of focal lengths $(100,165,180$, and 200 $\mathrm{mm}$ ). Although custom tube lens assemblies are possible [33], we found that off-the-shelf 200-mm (Nikon Tube Lens \#58-520, Edmund Optics) and 165-mm (TTL165-A, Thorlabs) tube lenses provide a magnification of $\sim 1.212 \mathrm{X}$, which matches the requirement for Nikon. Therefore, we decided to select a 20X Nikon objective for 02. To avoid the need for a cover glass, we narrowed our selection to Nikon objectives designed for use without a cover glass. This yielded one option, the LU (now TU) Plan Fluor EPI 20X $(N A=0.45)$. We chose this objective for the 02 in the current system.

To remotely correct for spherical aberrations introduced by the index mismatch of the specimen holder, it would also be possible to use a Nikon objective with a correction collar. There are two options, the CFI S Plan Fluor ELWD 20XC (NA = 0.45) with a correction collar for a cover glass of $t=0-2 \mathrm{~mm}$, and CFI S Plan Fluor LWD 20XC $(\mathrm{NA}=0.70)$ with a correction collar for a cover glass of $t=0-1.8 \mathrm{~mm}$. Both objectives could be explored in a future design (although spherical aberrations were not found to be an issue in the current design). In the case of the CFI S Plan Fluor LWD 20XC, the full 0.483 NA of our 01 objective could be transmitted to the remote focus, unlike the chosen LU Plan Fluor EPI 20X objective or CFI S Plan Fluor ELWD 20XC objective, which both clip things down to 0.45 NA.

The goal of $\mathrm{O} 3$ is to maximize light collection when tilted at the angle required to orthogonally image a remote version of the oblique light sheet within the specimen. As mentioned previously, in a singleobjective light-sheet design, the light sheet angle would be limited by our chosen 01 to a maximum of $28.9 \mathrm{deg}$. This would require 03 to be tilted by at least $61.1 \mathrm{deg}$. At this extreme tilt angle, one way to prevent light loss is through a custom solid- or liquid-immersion objective [32, 33]. However, a benefit of our NODO design is that the crossing angle can be increased to $45 \mathrm{deg}$, which reduces the tilt of 03 to 45 deg. At this tilt angle, an objective with NA $=0.95$ is able to capture light up to NA $=0.45$ and provide NAmaximized imaging. Therefore, we opted to use a NA $=0.95$ air objective for our 03 . This use of a tertiary air objective makes alignment more straightforward and stable than in the case of a solid- or liquidimmersion objective [32,33]. This is especially the case for liquid-immersion objectives, where there may be evaporation or leakage of a liquid medium over time.

In terms of selecting an optimal 03 for NA-maximized imaging, we only considered objectives from Olympus and Nikon for the same reasons as mentioned previously. Both companies offer two types of objectives with NA $=0.95$. These include $40 \mathrm{X}$ life-science objectives with a correction collar for cover- 
glass thicknesses ranging from $t=0.11-0.23 \mathrm{~mm}$, and 50X metrology objectives for imaging with no cover glass. Although it would be possible to permanently align or adhere a cover glass to the 40X objectives, we decided to select the CF IC EPI Plan Apo 50X objective from Nikon for our 03 for simplicity and ease-of-use. When combined with a 100-mm tube lens (TTL100-A, Thorlabs), our NODO imaging path provides a total magnification of $25 \times n$. This yields a sampling rate of $\sim 2.71$ (slightly better than Nyquist) when using a sCMOS camera with pixels spaced by $6.5 \mu \mathrm{m}$ (pco.edge 4.1, PCO Tech). The corresponding FOV is $\sim 0.53 \mathrm{~mm} / n$ (FOV $=0.40-0.34 \mathrm{~mm}$ when $n=1.33-1.56)$, which is not clipped by the $0.40-\mathrm{mm}$ FOV of the $50 \mathrm{X}$ objective. In this configuration, the back aperture of the illumination objective is filled, yielding an illumination NA of $\sim 0.10 \times n$. This corresponds to a confocal parameter of $\sim 40-50 \mu \mathrm{m}$ and a pixel height of $\sim 256$ pixels for each raw camera image. This also corresponds to an axial re-focusing range that is well within the range of operation for an idealized objective, as specified by Botcherby et al. [28]. The pixel width of each raw camera frame is the full 2048 pixels of the sCMOS camera, corresponding to a FOV of $\sim 0.34-0.40 \mathrm{~mm}$.

While the above 03 selection provides NA-maximized imaging, our 01 also provides a 1-mm FOV to enable FOV-maximized imaging (with slightly worse resolution). To achieve this, $\mathrm{O} 3$ can be changed to a 20X objective with a matched 1-mm FOV. To minimize light loss, 20X objectives with the highest NA were considered. For both Olympus and Nikon, the upper limit is NA $=0.75$ (although the recent UPLXAPO20X objective from Olympus offers $N A=0.80$ ). In a single-objective light-sheet configuration, the light sheet

angle in the specimen would be limited by the NA of 01 , requiring a larger remote tilt angle, which for this particular 03 would result in an effective NA $\sim 0.12 \times n$. In contrast, with our NODO configuration this results in an effective NA $\sim 0.26 \times n$. This reduced effective NA (in contrast to the NA-maximized case) enables close-to-Nyquist sampling of a 1-mm FOV with the same sCMOS chip as described earlier (with $2048 \times 2048$ pixels). To match parfocal lengths and thus enable a quick interchange of objectives, we selected the Nikon CFI Plan Apo Lambda 20X for our FOV-maximized 03 objective. When paired with the same 100-mm tube lens (TTL100-A, Thorlabs), this provides a total magnification of $10 \times n$, which yields a near-Nyquist sampling rate of 2.02. The effective FOV is $\sim 1.33 \mathrm{~mm} / n$ (FOV $=1.00-0.85 \mathrm{~mm}$ when $n=$ 1.33-1.56), which is not clipped by the $1.00 \mathrm{~mm}$ FOV of the 20X objective (03). The physical layout, ZEMAX model, and objective options for this FOV-maximized NODO imaging configuration are shown in Supplementary Fig. 11. See Supplementary Note 2 for more discussion of NA-versus FOV-maximized imaging. As mentioned previously, an alternative design could use the CFI S Plan Fluor LWD 20XC and a customized 03 to make use of the full 0.483 NA of our 01 (see Supplementary Fig. 12 and

\section{Supplementary Note 5).}

\section{ODO optical path}

The physical layouts and ZEMAX models of the ODO imaging configurations are shown in Supplementary Fig. 13-14. While the NODO imaging path can provide sub-micrometer resolution, the FOV of the multi-immersion primary objective (01) is restricted to $1 \mathrm{~mm}$, which is insufficient for fast mesoscopic imaging. This tradeoff between NA and FOV is standard across all currently available microscope objectives (e.g., no current clearing-compatible objectives can simultaneously offer sub- 
micrometer resolution over a mesoscopic FOV) (Supplementary Fig. 8) [49]. Therefore, in our hybrid system, we achieve low-resolution imaging with a second independent ODO imaging path.

The ODO collection path uses the same objective as the illumination optical path (TL2X-SAP, Thorlabs). The objective is similarly used in conjunction with a SIMlens (fabricated by BMV Optical), which provides multi-immersion performance and prevents axial chromatic aberrations in the ODO imaging path [26]. In addition, the SIMlens increases the NA of the collection path by a factor of $n$. This yields an effective NA $=0.10 \times n$.

The current set point of the ODO collection path lies between the NA- and FOV-maximized imaging extremes and uses a tube lens with a 200-mm focal length (TTL200-A, Thorlabs). This provides a magnification of $2 \times n$ and an effective FOV of $6.6 \mathrm{~mm} / n$, which undersamples the collection NA of the system when using a sCMOS camera with a pixel spacing of $6.5 \mu \mathrm{m}$ (pco.edge 4.1, PCO Tech, $2048 \mathrm{x}$ 2048 pixels). In this configuration, the back aperture of the illumination objective is underfilled to yield an illumination NA of $\sim 0.04 \mathrm{x} n$. This results in a confocal parameter of $\sim 250-300 \mu \mathrm{m}$, which corresponds to a digital image height of $\sim 128$ pixels for each raw camera image. The width (in pixels) of each raw camera image is the full 2048 pixels of the sCMOS camera, corresponding to a distance of $\sim 4.25-5 \mathrm{~mm}$ in the sample. This combination of illumination and collection NA provides near-isotropic resolution (Supplementary Fig. 16Error! Reference source not found.).

The magnification of the ODO imaging path is easily adjusted by changing the tube lens. NA-maximized imaging is achieved using a tube lens with a 400-mm focal length (AC508-400-A, Thorlabs). This provides a magnification of $4 \mathrm{x} n$ and an effective FOV of $3.3 \mathrm{~mm} / n$, which corresponds to a nearNyquist sampling rate of $\sim 2.1$ when using a sCMOS camera with a pixel spacing of $6.5 \mu \mathrm{m}$. FOVmaximized imaging is achieved using a tube lens with a 100-mm focal length (TTL100-A, Thorlabs). This provides a magnification of $1 \times n$ and an effective FOV of $13.33 \mathrm{~mm} / n$. See Supplementary Note 2 for more discussion of NA-versus FOV-maximized imaging modes.

\section{Image acquisition and post processing}

Image strips are collected with a combination of stage-scanning and lateral/vertical tiling. The stagescanning firmware is used to send a TTL SYNC trigger signal from the XY stage to the sCMOS camera for reproducible initiation of each imaging strip. After triggering, the camera is set to free-running mode and acquires the desired number of frames for a given image-strip length (as the sample is scanned by the stage at a constant velocity), with a spacing between adjacent frames that is identical to the sampling pitch of the raw camera images. For each raw frame, the camera uses the standard rolling shutter, where the shutter rolls from the center of the camera chip in both directions, as opposed to the light-sheet readout mode, where the shutter rolls from the top to the bottom of the camera chip. Using the standard rolling shutter, the rolling directions are oriented along the light sheet propagation direction. This orientation allows the imaging speed to be increased when the pixel height of each raw frame is cropped to match the confocal parameter of the illumination light sheet. 
At the start of each exposure, the camera sends a trigger to an analog output DAQ card (PCle-6738, National Instruments). The DAQ card then sends output voltages to the lasers, galvos, and ETL (for alignment only, not axial sweeping). To reduce motion blur, the lasers and galvos are triggered with a delay to only illuminate once the shutter has rolled across the full pixel height of the camera frame, resulting in a strobing effect. For the 128- or 256-pixel height of the ODO and NODO paths respectively, the rolling time, $t_{\text {roll }}$, is 0.625 and $1.25 \mathrm{~ms}$. The exposure time, $t_{\text {exp }}$, is set to $3 \times t_{\text {roll, }}$, resulting in a total exposure time, $t_{\text {tot }}$ of $4 \times t_{\text {roll, }}$ or 2.5 and $5 \mathrm{~ms}$ for the ODO and NODO paths. This corresponds to a data rate that is $1 / 4$ the maximum data rate of the SCMOS camera. The lateral scanning mirror is actuated with a sawtooth waveform and completes a single period within the total exposure time, $t_{\text {tot }}$ of the raw camera frame, corresponding to a frequency of $\sim 400$ and $\sim 200 \mathrm{~Hz}$ for the ODO and NODO paths. The ETL and second mirror are set to a pre-calibrated DC voltage for the entire image strip to yield an in-focus light sheet that is axially aligned to the center of the camera chip.

Raw camera frames are streamed from the camera to RAM and subsequently saved directly as a single HDF5 file and XML file with the associated metadata, which is suitable for immediate processing with BigStitcher via ImageJ [50,51]. This involves on-the-fly saving of down-sampled copies of the image strip $(2 x, 4 x, 8 x$, and 16x) in the hierarchical HDF5 file format. Additionally, the GPU-based B3D compression filter can be optionally added to the HDF5 writing process to yield 5-10X compression with negligible loss of usable information content [52]. However, both processing steps slow down the net data rate from RAM to disk. On-the-fly saving of down-sampled copies of the image strips slows the data rate by $\sim 2 x$, and the B3D algorithm slows down the process by another $\sim 2 x$. This net speed reduction of $\sim 4 x$ motivated our selection of the camera frame rate mentioned previously. For our experiments, this reduction in post-processing times and data-storage requirements are worth the reduction in imaging speed. However, it is important to note that the imaging speeds could be increased by $2 x$ if the aforementioned processing steps are omitted from the acquisition procedure, in which case the imaging speed would become limited by the duty cycle of the illumination strobing, where $t_{\exp }=t_{\text {roll }}$. The imaging speeds quoted in Fig. $1 \mathrm{~g}$ assume the $4 \mathrm{x}$-reduced data rate.

A 15\% overlap is used for both vertical and lateral tiling. Different wavelength channels are acquired sequentially. For each image strip "tile" that is acquired by laterally scanning the specimen in the $y$ direction, all channels are acquired by cycling through various laser/filter combinations and re-scanning that image strip for each laser/filter setting, before moving to the next tile position. When tiling vertically, the laser power is increased with depth per a user-defined exponential relationship, $P=P_{0} \times \exp (z / \mu)$, to account for the attenuation of the illumination light sheet as it penetrates deeper into the specimen (typically $\mu=5-20 \mathrm{~mm}^{-1}$ ). Finally, if desired, all of the imaging tiles can be aligned and fused into one contiguous 3D image as an HDF5 or TIFF file output using BigStitcher [50]. The entire image acquisition is controlled using a custom-written Python program that is available from the authors upon request.

\section{Computer hardware}


During acquisition, the images are collected by a dedicated custom workstation (Puget Systems) equipped with a high-specification motherboard (Asus WS C422 SAGE/10G), processor (Intel Xeon W2145 3.7GHz 8 Core $11 \mathrm{MB} 140 \mathrm{~W}$ ), and $256 \mathrm{~GB}$ of RAM. The motherboard houses several PCle cards, including 2 CameraLink frame grabbers (mEIV AD4/VD4, Silicon Software) for streaming images from the camera, a DAQ card (PCle-6738, National Instruments) for generating analog output voltages, a 10G SFP + network card (StarTech), and a GPU (TitanXP, NVIDIA). Datasets are streamed to a local 8 TB U.2 drive (Micron) that is capable of outpacing the data rates of the microscope system. Data is then transferred to a mapped network drive located on an in-lab server (X11-DPG-QT, SuperMicro) running 64bit Windows Server, equipped with 768 GB RAM and TitanXP (NVIDIA) and Quadro P6000 (NVIDIA) GPUs. The mapped network drive is a direct-attached RAID6 storage array with $15 \times 8.0$ TB HDDs. The RAID array is hardware based and controlled by an external 8-port controller (LSI MegaRaid 9380-8e $1 \mathrm{~GB}$ cache). Both the server and acquisition workstation are set with jumbo frames (Ethernet frame), and parallel send/receive processes matched to the number of computing cores on the workstation (8 physical cores) and server (16 physical cores), which reliably enables $\sim 1.0 \mathrm{~GB} \mathrm{sec}^{-1}$ network-transfer speeds.

\section{Preparation of ECi-cleared mouse brain}

Labeling and clearing was carried out as previously described [43]. For sparse labeling, a Slc17a7-Cre mouse (8 weeks old, female) received a systemic injection, via the retro-orbital sinus, of a mixture of Credependent Tet transactivator (PHP-eB-Syn-Flex-TRE-2x-tTA) and a reporter virus (PHP-eB-CAG-TRE3xGFP) $[43,53]$. High-titer (>10 $12 \mathrm{GC} / \mathrm{mL}$ ) viruses were obtained from the Janelia Research Campus Molecular Biology Core and diluted in sterile water when necessary.

Transfected mice were anesthetized with an overdose of isoflurane and then transcardially perfused with a solution of PBS containing $20 \mu \mathrm{g} / \mathrm{mL}$ heparin (Sigma-Aldrich \#H3393) followed by a $4 \%$ paraformaldehyde solution in PBS. Brains were extracted and post-fixed in $4 \%$ paraformaldehyde at $4{ }^{\circ} \mathrm{C}$ overnight (12-14 hrs) and washed in PBS to remove all traces of excess fixative (PBS changes were performed at $1 \mathrm{hr}, 6 \mathrm{hr}, 12 \mathrm{hr}$, and 1 day).

For amplification by immuno-labeling, brains were delipidated with a modified Adipo-Clear protocol [54]. Brains were washed with a methanol gradient series $(20 \%, 40 \%, 60 \%, 80 \%$, Fisher \#A412SK) in B1n buffer $\left(\mathrm{H}_{2} \mathrm{O} / 0.1 \%\right.$ Triton X-100/0.3 M glycine, $\mathrm{pH} 7 ; 4 \mathrm{~mL} /$ brain; one hr / step). Brains were then immersed in $100 \%$ methanol for $1 \mathrm{hr}, 100 \%$ dichloromethane (Sigma \#270997) for $1.5 \mathrm{hrs}$, and three times in 100\% methanol for $1 \mathrm{hr}$. Samples were then treated with a reverse methanol gradient series $(80 \%, 60 \%, 40 \%$, $20 \%$ ) in B1n buffer for 30 min each. All procedures were performed on ice. Samples were washed in B1n buffer for one hr and left overnight at room temperature; and then again washed in PTxwH buffer (PBS/0.1\% Triton X-100/0.05\% Tween $20 / 2 \mu \mathrm{g} / \mathrm{mL}$ heparin) with fresh solution after one and two hrs and then left overnight.

After delipidation, selected samples were incubated in primary antibody dilutions in PTxwH for 14 days on a shaker (1:1000, anti-GFP, Abcam, \#ab290). Samples were sequentially washed in $25 \mathrm{~mL}$ PTxwH for 
$1,2,4,8$, and three times for $24 \mathrm{hrs}$. Samples were incubated in secondary antibody dilutions in PTxwH for 14 days (1:600, AlexaFluor ${ }^{\circledR} 488$ conjugated donkey-anti-rabbit IgG) and washed in PTxwH similar to descriptions above. Finally, the tissue was dehydrated in ethanol grades $(25,50,75,100 \%)$ for $8 \mathrm{hr}$ per grade. The $100 \%$ grade was repeated to ensure removal of all water from the tissue. Finally, the tissue was cleared in ethyl-cinnamate (Sigma-Aldrich \#112372) for $8 \mathrm{hr}$ before imaging. All experimental protocols were conducted according to the National Institutes of Health guidelines for animal research and were approved by the Institutional Animal Care and Use Committee at Howard Hughes Medical Institute, Janelia Research Campus.

\section{Preparation of MDA-231/OS-RC-2 and aSMA-labeled mouse brains}

Human breast cancer cells, MDA-MB-231-5a-D (MDA-231) are highly metastatic clones from MDA-MB231 [55]. Human renal cell carcinoma, OS-RC-2 were kindly provided by Prof. Tatsuro Irimura (Juntendo University, Japan). MDA-231 cells were cultured in Dulbecco's modified eagle's medium (DMEM) supplemented with $10 \%$ fetal bovine serum (FBS), $100 \mathrm{U} / \mathrm{ml}$ penicillin and $100 \mu \mathrm{g} / \mathrm{ml}$ streptomycin as previously described [55]. OS-RC-2 cells were maintained in RPMI 1640 containing $10 \%$ FBS and penicillin/streptomycin [56].

To establish cancer cells stably expressing firefly luciferase and mCherry under the EF-1 promoter, a lentiviral expression system was used (kindly provided by Dr Hiroyuki Miyoshi, deceased, formerly Keio University, Tokyo, Japan) as described previously (Miyoshi et al., Proc. Natl. Acad. Sci., 1997). Briefly, 293FT cells were transfected with a vector construct encoding the expression protein, VSV-G, a Revexpressing construct (pCMV-VSV-G-RSV-Rev), and a packaging construct (pCAG-HIVgp). The culture supernatants containing viral particles were collected and used as lentiviral vectors.

BALB/c-nu/nu mice (4 weeks old, female) were purchased from Japan SLC (Shizuoka, Japan). All experiments were approved and carried out according to the Animal Care and the Use Committee of the Graduate School of Medicine, The University of Tokyo. For developing experimental brain metastasis models by intracardiac (i.c.) inoculation, BALB/c-nu/nu mice were injected with MDA-231 or OS-RC-2 cells $\left(5 \times 10^{5}\right.$ cells /mouse) by puncture into the left ventricle of the heart.

Clearing, 3D staining, and imaging of whole mouse brain samples was performed with CUBIC clearing and CUBIC-HistoVision (CUBIC-HV) staining protocols [7, 10]. An updated CUBIC-HV staining protocol was used (HV1.1, commercialized by CUBICStars Co. and Tokyo Chemical Industry (TCI), TCl \#C3709, \#C3708). In brief, the PFA-fixed whole mouse brains were treated with CUBIC-L for 4 days at $37^{\circ} \mathrm{C}$, washed with PBS, stained with SYTOX-G (1/2500) in CUBIC-HV nuclear-staining buffer (included in TCI \#C3709) for 5 days at $37^{\circ} \mathrm{C}$, and then washed / immersed in $50 \%$ and $100 \%$ CUBIC-R + for 1 day and 3 days, respectively, at room temperature. We used CUBIC-R+(M) [45 wt\% of antipyrine (TCl \#D1876), $30 \mathrm{wt} \%$ of $\mathrm{N}$-methylnicotinamide ( $\mathrm{TCl} \# \mathrm{M} 0374)$, and $0.5 \%(\mathrm{v} / \mathrm{w}) \mathrm{N}$-butyldiethanolamine ( $\mathrm{TCl}$ \#B0725), adjusted to $\mathrm{pH} \sim 10]$ for brains containing cancer cells, and CUBIC-R+(N) [45 wt\% of antipyrine, $30 \mathrm{wt} \%$ of 
nicotinamide (TCl \#N0078), and 0.5\% (v/w) N-butyldiethanolamine, adjusted to $\mathrm{pH} \sim 10$ ] for the aSMA immunostained brain, respectively.

For the whole mouse brain immunostained with anti-a-SMA (Sigma, \#A5228) antibodies, the brain was subjected to CUBIC-HV immunostaining. The brain was first treated with $3 \mathrm{mg} / \mathrm{mL}$ hyaluronidase in CAPSO buffer $(\mathrm{pH} 10)$ for $24 \mathrm{~h}$ at $37^{\circ} \mathrm{C}$. After washing with hyaluronidase wash buffer [50 mM Carbonate buffer, $0.1 \%(\mathrm{v} / \mathrm{v})$ Triton X-100, 5\% (v/v) Methanol, and 0.05\% NaN3] and HEPES-TSC buffer [10 mM HEPES buffer, $\mathrm{pH} 7.5,10 \%$ (v/v) Triton X-100, $200 \mathrm{mM} \mathrm{NaCl}, 0.5 \%$ (w/v) casein, and $0.05 \% \mathrm{NaN} 3]$, the brain was immersed in $500 \mu \mathrm{L}$ of HEPES-TSC buffer containing a primary antibody ( $6 \mu \mathrm{g}$ for anti-a-SMA), a secondary Fab fragment (FabuLight, Jackson immunolab, Alexa Fluor ${ }^{\circledR} 594$ Goat Anti-Mouse IgG1 \#115-587-185, Alexa Fluor® 594 Goat Anti-Rabbit lgG \#111-587-008, Alexa Fluor® 594 Goat Anti-Mouse IgG2a \#115-587-186, 1:0.75 of weight ratio), and 3D immunostaining additive (1x) (included in TCl \#C3708). Then, the sample was incubated with gentle shaking for 10 days at $32^{\circ} \mathrm{C}$. After staining, the sample was additionally incubated in the same buffer for 1 day at $4^{\circ} \mathrm{C}$ to stabilize the Fab binding. Then, the sample was washed and post-fixed according to the protocol of CUBIC-HV immunostaining kit (TCI \#C3708) before being index-matched with CUBIC-R+. The cleared sample was embedded in CUBIC-Ragarose for imaging and storage [57]. This animal experimental procedures and housing conditions of the animals were approved by the Animal Care and Use Committees of the Graduate School of Medicine of the University of Tokyo.

\section{Statistics and reproducibility}

For statistical analysis, we reported the mean, standard deviation, and number of observations. For repeatability, only one sample was used for each imaging experiment, unless otherwise noted.

\section{Declarations}

\section{Data Availability}

The customized ZEMAX files are available as Supplementary Data. Imaging datasets are available from the authors upon request.

\section{Code Availability}

The simulation codes used to model the lateral and axial resolution of the various microscope architectures is available on GitHub and as Supplementary Code. The acquisition software code for the microscope is available from the authors upon request.

\section{Acknowledgements}

We would like to thank Andrew York and Alfred Millet-Sikking for discussions regarding oblique planar microscopy, remote focus imaging, and alignment procedures. We would also like to thank Philip (Rusty) Nicovich for discussions on the NODO architecture, and Jon Daniels for discussions and his development of the multi-immersion objective. We would also like to thank Chika Shimizu (RIKEN BDR) for the support 
of preparing CUBIC-cleared and stained specimens and Kohei Miyazono (The University of Tokyo) for the discussion and support of brain metastasis experiments. This work was funded in part by the National Institutes of Health (NIH) K99 CA240681 (Glaser), R01CA244170 (Liu), R01EB031002 (Liu), R01GM079712 (Imaizumi), R01DK107436 (Xin), R01DK092202 (Xin); Department of Defense (DoD) Prostate Cancer Research Program (PCRP) W81XWH-18-10358 (Liu and True), W81XWH-19-1-0589 (Reder), W81XWH-20-1-0039 (Wei), and Prostate Cancer Young Investigator Award (Reder); National Science Foundation (NSF) Graduate Research Fellowship DGE-1762114 (Barner and Bishop); NSF 1934292 HDR: I-DIRSE-FW (Liu and Serafin); Washington Research Foundation Postdoctoral Fellowship (Stoltzfus); Science and Technology Platform Program for Advanced Biological Medicine by the Japan Agency for Medical Research and Development (AMED) JP21am0401011 (Ueda), ERATO by Japan Science and Technology Agency (JST) JPMJER2001 (Ueda), HFSP Research Grant Program RGP0019/2018 (Ueda), AMED-PRIME JP21gm6210027 (Susaki), Grants-in-Aid for Scientific Research on Innovative Areas (JSPS KAKENHI grant) 17H06328 (Susaki), Grants-in-Aid for Scientific Research on Innovative Areas (JSPS KAKENHI grant) 20K1612 (Kubota). Work in the Murawala laboratory is supported by grants from NIH-COBRE (5P20GM104318-08) and DFG (429469366).

\section{Author Contributions}

A.K.G. and J.T.C.L. conceived of and designed the microscope system. P.R.N. provided feedback on the system design and its potential applications. A.K.G., K.W.B., R.B.S., and G.G. performed simulations of the microscope. A.K.G. fabricated the microscope system with help from L.A.B. E.A.S. and H.R.U. provided and prepared the immunostained CUBIC-cleared mouse brains. E.A.S., S.I.K., and H.R.U. prepared and provided the metastatic mouse brains. J.C. and K.S. prepared and provided the mouse brain. P.B., E.T., and R.C.R. provided the mouse brain with preparation by A.K.G. The human brain slice was prepared by A.K.G. H.L. and L.A.G.L. provided quantitative analysis of the high-resolution images. Y.Y. and H.Z. provided and prepared the PEGASOS-cleared mouse brain. E.K.N., B.J.B., and J.S. provided and prepared the SHIELD-cleared mouse embryos. H.H., N.P.R., and L.D.T. provided and prepared the ECi-cleared human prostate tissue. J.J.W., R.S., E.S., C.R.S., and M.Y.G. provided and prepared the Ce3D-cleared mouse lymph node. X.W. and L.X. provided and prepared the iDISCO-cleared mouse prostate. A.K.H. and T.I. provided and prepared the ClearSee-processed Arabidopsis plant and M.P., P.M., and H.U.D. provided and prepared the DEEP-Clear-processed Axolotl. A.K.G. and J.T.C.L. led the writing of the manuscript. All authors contributed to the manuscript.

\section{Competing Interests}

A.K.G., N.P.R., L.D.T., and J.T.C.L. are co-founders and shareholders of Lightspeed Microscopy Inc.

\section{Corresponding Author}

Correspondence to Adam K. Glaser or Jonathan T.C. Liu

\section{References}


1. Chung, K., et al., Structural and molecular interrogation of intact biological systems. Nature, 2013. 497(7449): p. 332-7.

2. Tanaka, N., et al., Whole-tissue biopsy phenotyping of three-dimensional tumours reveals patterns of cancer heterogeneity. Nature Biomedical Engineering, 2017. 1(10): p. 796.

3. Susaki, E.A., et al., Advanced CUBIC protocols for whole-brain and whole-body clearing and imaging. Nature Protocols, 2015. 10: p. 1709-1727.

4. Richardson, D.S. and J.W. Lichtman, Clarifying Tissue Clearing. Cell, 2015. 162(2): p. 246-57.

5. Pan, C., et al., Shrinkage-mediated imaging of entire organs and organisms using uDISCO. Nat Methods, 2016. 13(10): p. 859-67.

6. Renier, N., et al., iDISCO: a simple, rapid method to immunolabel large tissue samples for volume imaging. Cell, 2014. 159(4): p. 896-910.

7. Tainaka, K., et al., Chemical Landscape for Tissue Clearing Based on Hydrophilic Reagents. Cell Reports, 2018. 24(8): p. 2196-2210.e9.

8. Susaki, E.A., et al., Advanced CUBIC protocols for whole-brain and whole-body clearing and imaging. Nat Protoc, 2015. 10(11): p. 1709-27.

9. Chung, K. and K. Deisseroth, CLARITY for mapping the nervous system. Nat Methods, 2013. 10(6): p. 508-13.

10. Susaki, E.A., et al., Versatile whole-organ/body staining and imaging based on electrolyte-gel properties of biological tissues. Nature Communications, 2020. 11(1): p. 1982.

11. Jing, D., et al., Tissue clearing of both hard and soft tissue organs with the PEGASOS method. Cell Res, 2018. 28(8): p. 803-818.

12. Power, R.M. and J. Huisken, A guide to light-sheet fluorescence microscopy for multiscale imaging. Nature Methods, 2017. 14(4): p. 360.

13. Huisken, J. and D.Y.R. Stainier, Selective plane illumination microscopy techniques in developmental biology, in Development. 2009. p. 1963-75.

14. Dodt, H.-U., et al., Ultramicroscopy: three-dimensional visualization of neuronal networks in the whole mouse brain. Nature Methods, 2007. 4(4): p. 331-336.

15. Migliori, B., et al., Light sheet theta microscopy for rapid high-resolution imaging of large biological samples. BMC Biology, 2018. 16(1): p. 57.

16. Tomer, R., et al., Advanced CLARITY for rapid and high-resolution imaging of intact tissues. Nature Protocols, 2014. 9(7): p. 1682-97.

17. Voigt, F.F., et al., The mesoSPIM initiative: open-source light-sheet microscopes for imaging cleared tissue. Nature Methods, 2019. 16(11): p. 1105-1108.

18. Chakraborty, T., et al., Light-sheet microscopy of cleared tissues with isotropic, subcellular resolution. Nature Methods, 2019. 16(11): p. 1109-1113.

19. Dodt, H.-U., et al., Ultramicroscopy: three-dimensional visualization of neuronal networks in the whole mouse brain. Nat Methods, 2007. 4(4): p. 331-336. 
20. Kumar, A., et al., Dual-view plane illumination microscopy for rapid and spatially isotropic imaging. Nature Protocols, 2014. 9: p. 2555-2573.

21. Strnad, P., et al., Inverted light-sheet microscope for imaging mouse pre-implantation development. Nature Methods, 2015. 13: p. 139-142.

22. McGorty, R., et al., Open-top selective plane illumination microscope for conventionally mounted specimens. Optics Express, Vol. 23, Issue 12, pp. 16142-16153, 2015.

23. McGorty, R., D. Xie, and B. Huang, High-NA open-top selective-plane illumination microscopy for biological imaging. Optics Express, 2017. 25(15): p. 17798-810.

24. Glaser, A.K., et al., Light-sheet microscopy for slide-free non-destructive pathology of large clinical specimens. Nature Biomedical Engineering, 2017. 1(7): p. 0084.

25. Glaser, A.K., et al., Multi-immersion open-top light-sheet microscope for high-throughput imaging of cleared tissues. Nature Communications, 2019. 10(1): p. 1-8.

26. Barner, L.A., et al., Solid immersion meniscus lens (SIMlens) for open-top light-sheet microscopy. Optics Letters, 2019. 44(18): p. 4451-4454.

27. Barner, L.A., et al., Multi-resolution open-top light-sheet microscopy to enable efficient 3D pathology workflows. Biomedical Optics Express, 2020. 11(11): p. 6605-6619.

28. Botcherby, E.J., et al., An optical technique for remote focusing in microscopy. Optics Communications, 2008. 281(4): p. 880-887.

29. Dunsby, C., Optically sectioned imaging by oblique plane microscopy. Optics Express, 2008. 16(25): p. 20306-20316.

30. Voleti, V., et al., Real-time volumetric microscopy of in vivo dynamics and large-scale samples with SCAPE 2.0. Nature Methods, 2019. 16(10): p. 1054-1062.

31. Bouchard, M.B., et al., Swept confocally-aligned planar excitation (SCAPE) microscopy for high-speed volumetric imaging of behaving organisms. Nature Photonics, 2015. 9(2): p. 113-119.

32. Yang, B., et al., Epi-illumination SPIM for volumetric imaging with high spatial-temporal resolution. Nature Methods, 2019. 16(6): p. 501-504.

33. Millett-Sikking, A., et al. High NA single-objective light-sheet. 2019; Available from: https://andrewgyork.github.io/high_na_single_objective_lightsheet/index.html.

34. Kumar, M., et al., Integrated one- and two-photon scanned oblique plane illumination (SOPi) microscopy for rapid volumetric imaging. Optics Express, 2018. 26(10): p. 13027-13041.

35. Hoffmann, M. and B. Judkewitz, Diffractive oblique plane microscopy. Optica, 2019. 6(9): p. 5.

36. Sapoznik, E., et al., A versatile oblique plane microscope for large-scale and high-resolution imaging of subcellular dynamics. eLife, 2020. 9: p. e57681.

37. Yang, B., et al., High-Resolution, Large Imaging Volume, and Multi-View Single Objective Light-Sheet Microscopy. bioRxiv, 2021: p. 2020.09.22.309229.

38. Kumar, M., et al., Crossbill: an open access single objective light-sheet microscopy platform. bioRxiv, 2021: p. 2021.04.30.442190. 
39. Li, T., et al., Axial Plane Optical Microscopy. Scientific Reports, 2014. 4(1): p. 7253.

40. Kim, J., et al., Oblique-plane single-molecule localization microscopy for tissues and small intact animals. Nature Methods, 2019. 16(9): p. 853-857.

41. Bishop, K.W., A.K. Glaser, and J.T.C. Liu, Performance tradeoffs for single- and dual-objective opentop light-sheet microscope designs: a simulation-based analysis. Biomed Opt Express, 2020. 11(8): p. 4627-4650.

42. Economo, M.N., et al., A platform for brain-wide imaging and reconstruction of individual neurons. elife, 2016. 5: p. e10566.

43. Winnubst, J., et al., Reconstruction of 1,000 Projection Neurons Reveals New Cell Types and Organization of Long-Range Connectivity in the Mouse Brain. Cell, 2019. 179(1): p. 268-281.e13.

44. Kubota, S.I., et al., Whole-Body Profiling of Cancer Metastasis with Single-Cell Resolution. Cell Reports, 2017. 20(1): p. 236-250.

45. Dean, K., et al., Deconvolution-free Subcellular Imaging with Axially Swept Light Sheet Microscopy, in Biophys J. 2015. p. 2807-15.

46. Planchon, T.A., et al., Rapid three-dimensional isotropic imaging of living cells using Bessel beam plane illumination. Nat Methods, 2011. 8(5): p. 417-423.

47. Sparks, H., et al., Dual-view oblique plane microscopy (dOPM). Biomedical Optics Express, 2020. 11(12): p. 7204-7220.

48. Keller, P.J., et al., Fast, high-contrast imaging of animal development with scanned light sheet-based structured-illumination microscopy. Nat Methods, 2010. 7(8): p. 637-42.

49. Yueqian, Z. and G. Herbert, Systematic design of microscope objectives. Part I: System review and analysis. Advanced Optical Technologies, 2019. 8(5): p. 313-347.

50. Hörl, D., et al., BigStitcher: reconstructing high-resolution image datasets of cleared and expanded samples. Nature Methods, 2019. 16(9): p. 870-874.

51. Schindelin, J., et al., Fiji: an open-source platform for biological-image analysis. Nature Methods, 2012. 9(7): p. 676-682.

52. Balazs, B., et al., A real-time compression library for microscopy images. bioRxiv, 2017.

53. Chan, K.Y., et al., Engineered AAVs for efficient noninvasive gene delivery to the central and peripheral nervous systems. Nature Neuroscience, 2017. 20(8): p. 1172-1179.

54. Chi, J., et al., Three-Dimensional Adipose Tissue Imaging Reveals Regional Variation in Beige Fat Biogenesis and PRDM16-Dependent Sympathetic Neurite Density. Cell Metabolism, 2018. 27(1): p. 226-236.e3.

55. Ehata, S., et al., Transforming Growth Factor- $\beta$ Promotes Survival of Mammary Carcinoma Cells through Induction of Antiapoptotic Transcription Factor DEC1. Cancer Research, 2007. 67(20): p. 9694.

56. Nishida, J., et al., Epigenetic remodelling shapes inflammatory renal cancer and neutrophil-dependent metastasis. Nature Cell Biology, 2020. 22(4): p. 465-475. 
57. Matsumoto, K., et al., Advanced CUBIC tissue clearing for whole-organ cell profiling. Nature Protocols, 2019. 14(12): p. 3506-3537.

\section{Figures}

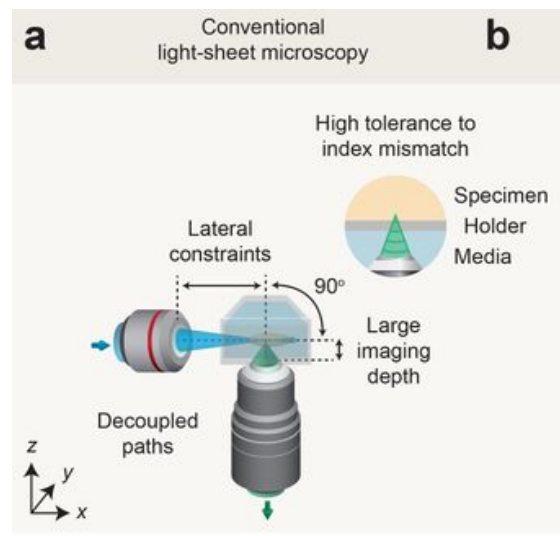

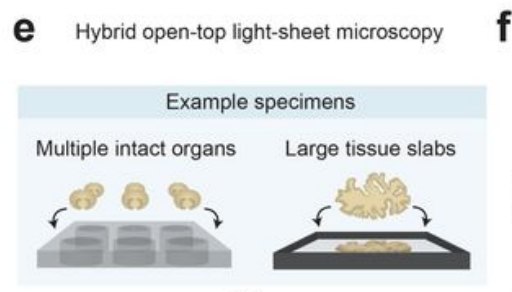

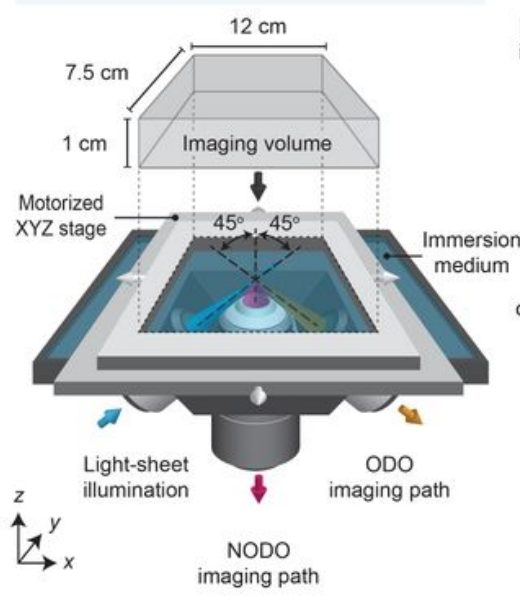

g

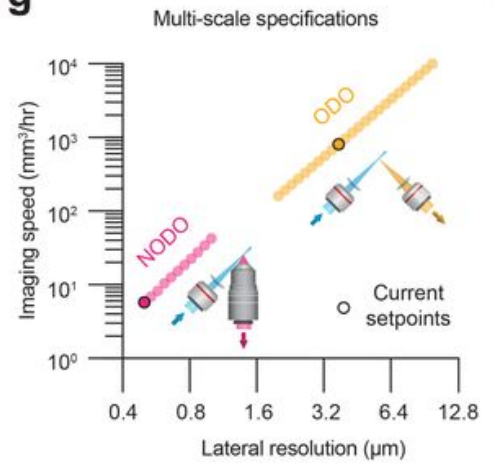

Orthogonal dual-objective (ODO)
open-top light-sheet microscopy Low tolerance to

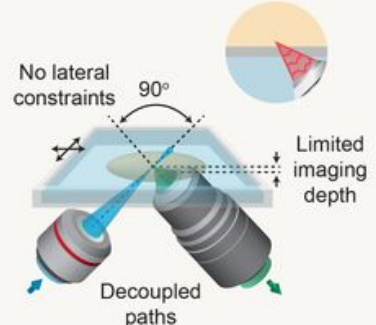
paths

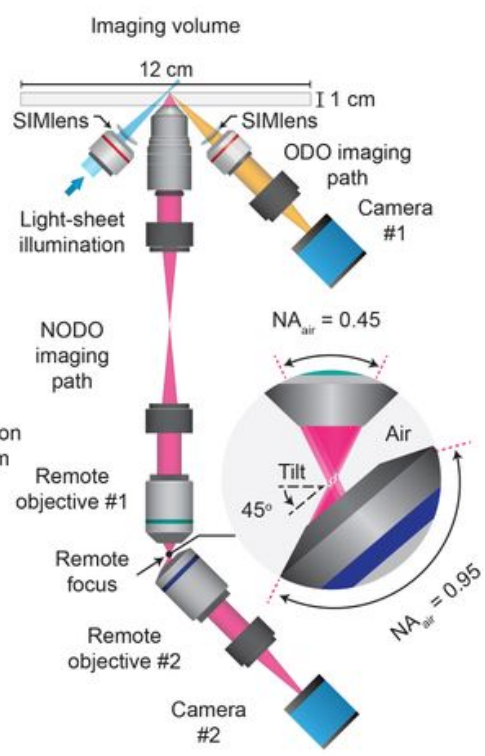

h

Point spread functions $\quad$ FWHM $(\mu \mathrm{m})$
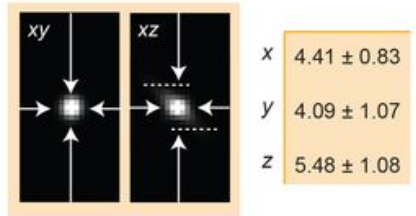

Figure 1

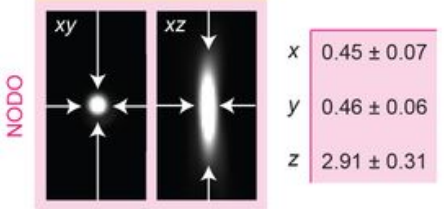

\section{C \\ Single-objective \\ open-top light-sheet microscopy}

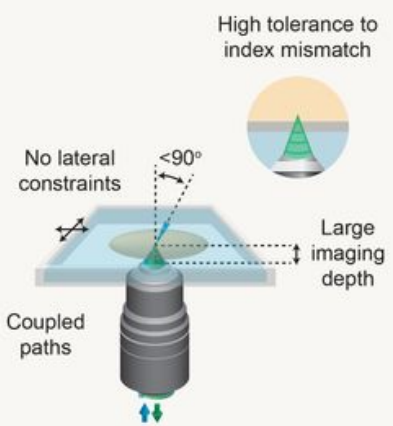

Non-orthogonal dual-objective (NODO) open-top light-sheet microscopy

\section{High tolerance to}

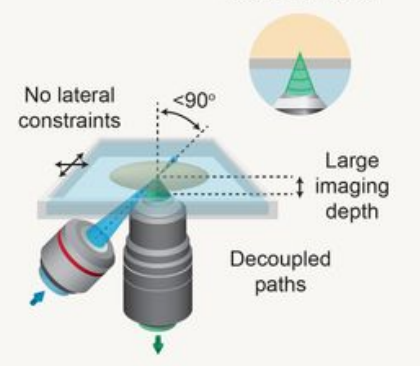

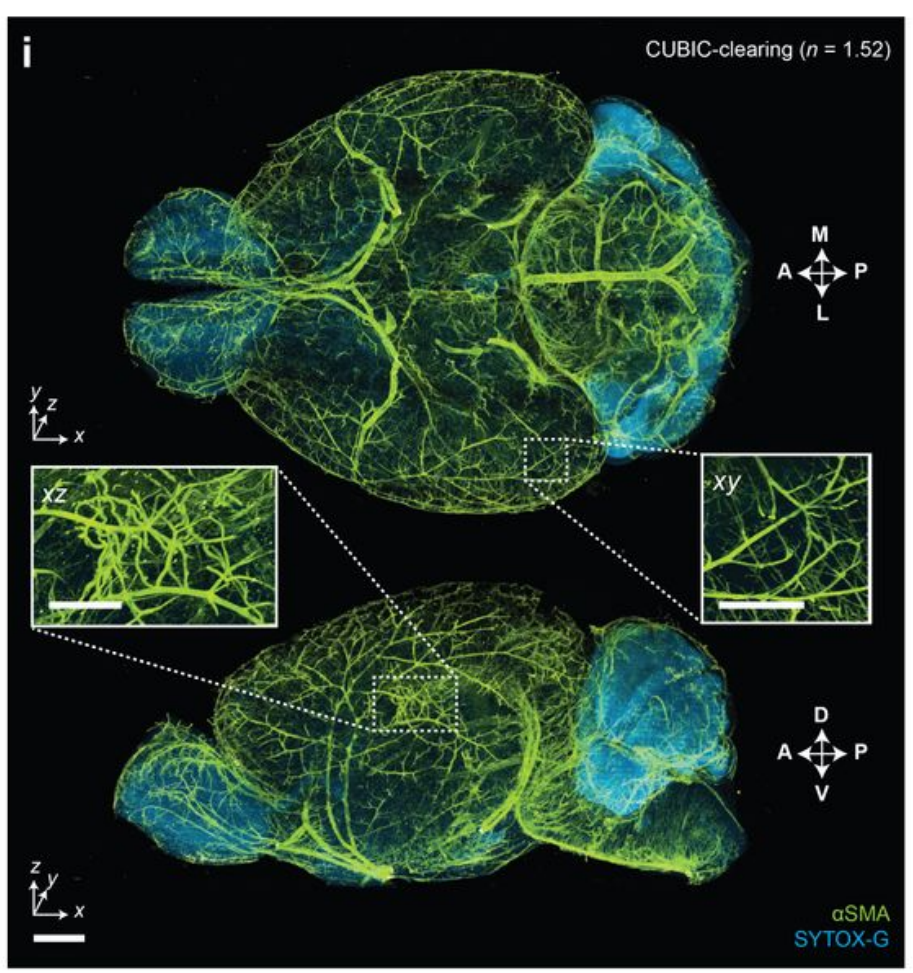

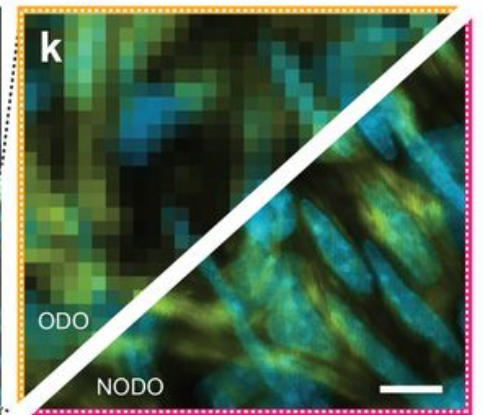


Hybrid open-top light-sheet (OTLS) microscopy. (a) Orthogonal dual-objective (ODO) light-sheet microscopes typically place one or more objectives in the horizontal plane of the specimen, which constrains the specimen size and prevents the use of standard modular sample holders. (b) ODO opentop light-sheet (OTLS) microscopy systems overcome this constraint by tilting the objectives and positioning them beneath a horizontal sample holder. However, a consequence of this is a limited imaging depth and low tolerance to refractive-index mismatch between the specimen and the sampleholder material (which can reduce ease-of-use). (c) Single-objective OTLS microscopy systems overcome both limitations. However, the use of a single objective for illumination and collection precludes broad multi-scale imaging and limits/couples the axial and lateral resolutions. (d) Non-orthogonal dualobjective (NODO) OTLS microscopy allows for laterally unconstrained imaging over the full working distance of a vertically oriented collection objective with high tolerance to index mismatch. The use of a separate objective for light-sheet illumination also enables more design flexibility to optimize axial and lateral resolutions. (e) The hybrid microscope architecture consists of three objectives, positioned below the specimen, mounted into a monolithic imaging chamber that is filled with an interchangeable immersion medium. One objective is used for light-sheet illumination, and the other two objectives are used for orthogonal dual-objective (ODO) and non-orthogonal dual-objective (NODO) collection. The ODO path provides fast meso-scale screening capabilities, and the NODO path enables targeted sub-

micrometer imaging. By using a motorized stage, tiled imaging is possible with both paths over a large 12 $x 7.5 \times 1 \mathrm{~cm}(X Y Z)$ imaging volume. This large imaging volume accommodates multiple intact cleared organs and large tissue slabs mounted in an array of specimen holders. The optical layout of the hybrid OTLS system is shown in (f). The ODO path uses a single tube lens to directly image the light sheet onto Camera \#1. The NODO path uses a remote focus system consisting of three objective-tube lens pairs to image the non-orthogonal light sheet onto Camera \#2. (g) In combination, the ODO and NODO paths enable imaging over a tunable lateral resolution range of $0.5-10.7 \mu \mathrm{m}$ at imaging speeds of $\sim 5 \mathrm{~mm} 3$ to $10 \mathrm{~cm} 3$ per hour. The current set points of the system are highlighted. (h) At these set points, the fullwidth half-maximum (FWHM) resolutions (xyz) for the ODO path are $4.09 \pm 1.07,4.41 \pm 0.83$, and $5.48 \pm$ $1.08 \mu \mathrm{m}$, and for the NODO path are $0.45 \pm 0.07,0.46 \pm 0.06$, and $2.91 \pm 0.31 \mu \mathrm{m}$ (mean \pm standard deviation). These values are measured from reflectance imaging of $150-\mathrm{nm}$ gold beads in an ECi-cleared agarose phantom (638-nm illumination). (i) Representative ODO imaging results of an entire intact CUBIC-cleared mouse brain with arterial (ASMA) and nuclear (SYTOX-G) staining. The ODO imaging path, with near-isotropic resolution, is able to clearly resolve vasculature in both the $x y$ and $x z$ planes (insets). (j-k) Targeted imaging of a sub-region centered on a branching arteriole using the NODO imaging path resolves individual smooth muscle cells and sub-nuclear features that are not resolved by ODO imaging. Scale-bar lengths are as follows: (i) $1 \mathrm{~mm}$ (insets, $500 \mu \mathrm{m}$ ), (j) $100 \mu \mathrm{m}$, and (k) $10 \mu \mathrm{m}$. All images are displayed without deconvolution. 

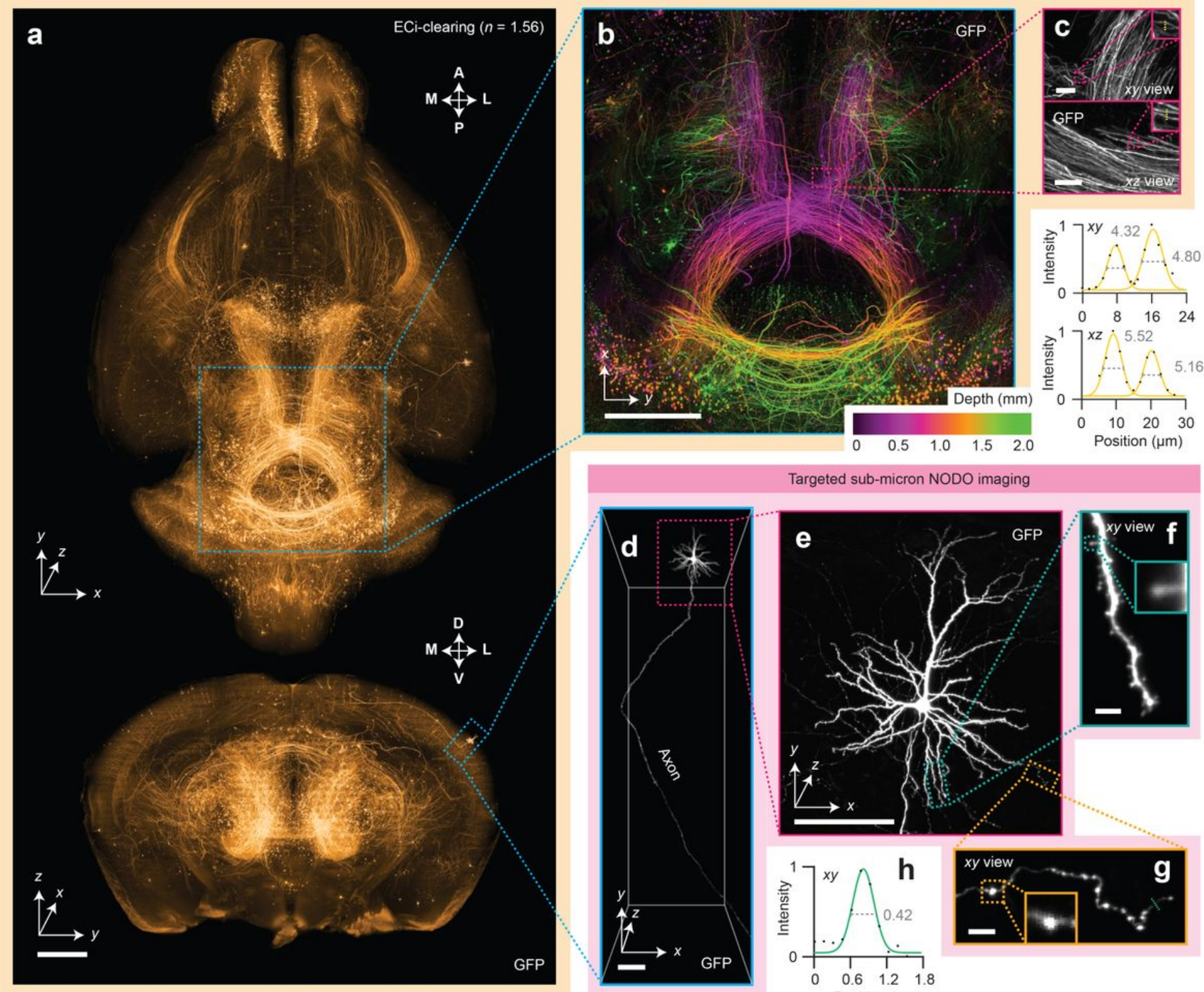

Targeted sub-micron NODO imaging
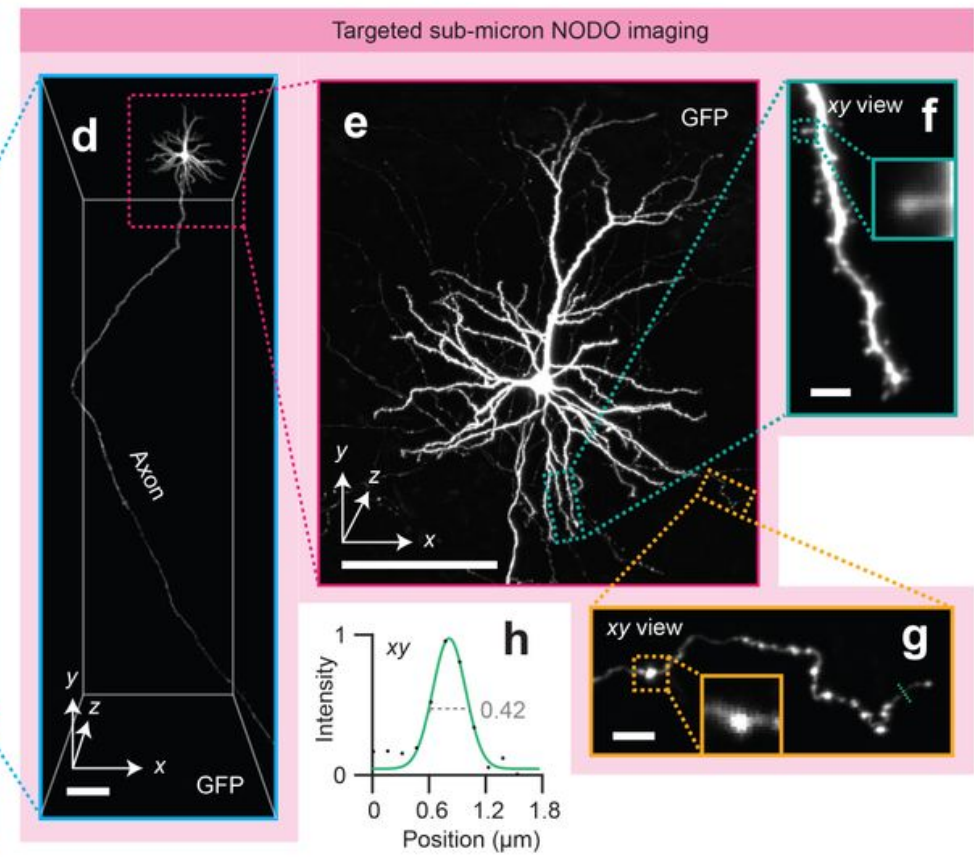

Figure 2

Fast meso-scale screening and targeted sub-micrometer imaging in cleared tissues. (a) Fast meso-scale screening is performed of an entire intact ECi-cleared Slc17a7-Cre mouse brain with brain-wide axonal projections. (b) A depth-coded region of interest shows dense projections in the midbrain. (c) xy and xz zoom-in views illustrate the near-isotropic resolution of the hybrid OTLS microscope. Line profiles through individual axons demonstrate an ODO lateral and axial resolution of 4-5 $\mu \mathrm{m}$ at a large depth within the cleared specimen. (d-e) Targeted sub-micrometer imaging is performed of a region of interest around a cortical pyramidal neuron. (f-g) Zoom-ins of a dendrite and axon demonstrate sufficient lateral resolution to visualize individual spines and varicosities. (h) A line profile through an individual axon demonstrates a NODO lateral resolution of $0.42 \mu \mathrm{m}$ within the cleared specimen. Scale-bar lengths are as follows: (a-b) $1 \mathrm{~mm},(\mathrm{c}) 10 \mu \mathrm{m},(\mathrm{d}-\mathrm{e}) 100 \mu \mathrm{m}$, and (f-g) $5 \mu \mathrm{m}$. All images are displayed without deconvolution. 


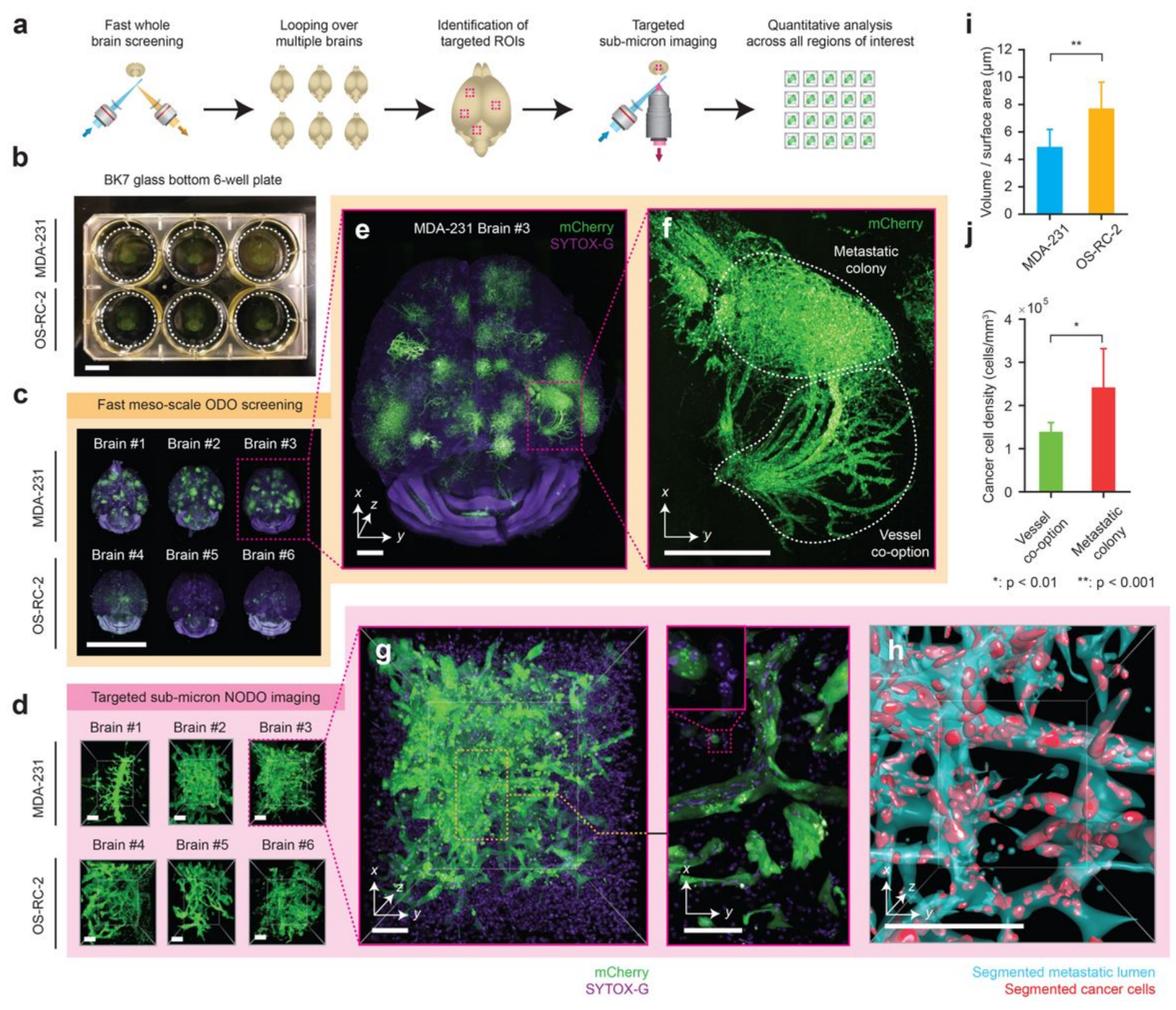

\section{Figure 3}

Multi-scale OTLS microscopy for quantitative analysis of brain metastases. (a) Hybrid OTLS microscopy workflow for multi-scale imaging of multiple specimens. (b) $\mathrm{N}=6$ whole mouse brains containing metastatic lesions from two different cancer cell lines (MDA-231 and OS-RC-2) were placed into a glassbottom 6-well plate and mounted onto the hybrid OTLS microscope system for multi-scale imaging. (c) The ODO imaging path was used to rapidly screen multiple intact mouse brains containing metastatic colonies. (d) $\mathrm{N}=34$ total metastatic regions of interest (ROI) across all brains were identified and subsequently imaged at sub-micrometer resolution using the NODO imaging path (only one ROI per brain is shown for illustrative purposes). Visual inspection of a single brain with MDA-231 metastases in (e) revealed multiple colonies distributed throughout the brain, with signs of vessel co-option ( $\mathrm{f}-\mathrm{g}$ ) that were not observed for OS-RC-2 metastases. (h) To quantify these phenotypic differences between the MDA-231 and OS-RC-2 metastases, the metastatic lumens (cyan) and cancer cells (red) were computationally 
segmented in all $\mathrm{N}=34$ ROls. Quantification of the resulting segmentation masks revealed statistically (two-sample t-test) different 3D growth patterns between the two cancer cell lines, consistent with a previous report (i-j) [44]. Error bars in ( $\mathrm{i}-\mathrm{j}$ ) denote standard deviation. Scale-bar lengths are as follows: (bc) $1 \mathrm{~cm}$, (d) $100 \mu \mathrm{m}$, (e) $1 \mathrm{~mm}$, (f) $500 \mu \mathrm{m}$, and (g-h) $100 \mu \mathrm{m}$. All images are displayed without deconvolution.

\section{Supplementary Files}

This is a list of supplementary files associated with this preprint. Click to download.

- supplementarymaterial.docx

- objectivedata.zip

- zemax.zip

- supplementaryvideo1.mp4

- supplementaryvideo2.mp4

- supplementaryvideo3.mp4

- supplementaryvideo4.mp4

- supplementaryvideo5.mp4

- supplementaryvideo6.mp4

- supplementaryvideo7.mp4

- supplementaryvideo8.mp4

- supplementaryvideo9.mp4

- supplementaryvideo10.mp4 